\title{
Vaccination Targeting a Surface Sialidase of $P$. acnes: Implication for New Treatment of Acne Vulgaris
}

\author{
Teruaki Nakatsuji ${ }^{1,2}$, Yu-Tsueng Liu ${ }^{3}$, Cheng-Po Huang ${ }^{2,3}$, Richard L. Gallo ${ }^{1,2}$, Chun-Ming Huang ${ }^{1,2,3,4 *}$
}

1 Division of Dermatology, Department of Medicine, University of California San Diego, San Diego, California, United States of America, 2 Veterans Affairs (VA) San Diego Healthcare Center, San Diego, California, United States of America, 3 Moores Cancer Center, University of California San Diego, San Diego, California, United States of America, 4 La Jolla Institute for Molecular Medicine, San Diego, California, United States of America

\begin{abstract}
Background: Acne vulgaris afflicts more than fifty million people in the United State and the severity of this disorder is associated with the immune response to Propionibacterium acnes ( $P$. acnes). Systemic therapies for acne target $P$. acnes using antibiotics, or target the follicle with retinoids such as isotretinoin. The latter systemic treatment is highly effective but also carries a risk of side effects including immune imbalance, hyperlipidemia, and teratogenicity. Despite substantial research into potential new therapies for this common disease, vaccines against acne vulgaris are not yet available.

Methods and Findings: Here we create an acne vaccine targeting a cell wall-anchored sialidase of $P$. acnes. The importance of sialidase to disease pathogenesis is shown by treatment of a human sebocyte cell line with recombinant sialidase that increased susceptibility to $P$. acnes cytotoxicity and adhesion. Mice immunized with sialidase elicit a detectable antibody; the anti-sialidase serum effectively neutralized the cytotoxicity of $P$. acnes in vitro and $P$. acnes-induced interleukin-8 (IL-8) production in human sebocytes. Furthermore, the sialidase-immunized mice provided protective immunity against $P$. acnes in vivo as this treatment blocked an increase in ear thickness and release of pro-inflammatory macrophage inflammatory protein (MIP-2) cytokine.
\end{abstract}

Conclusions: Results indicated that acne vaccines open novel therapeutic avenues for acne vulgaris and other $P$. acnesassociated diseases.

Citation: Nakatsuji T, Liu Y-T, Huang C-P, Gallo RL, Huang C-M (2008) Vaccination Targeting a Surface Sialidase of $P$. acnes: Implication for New Treatment of Acne Vulgaris. PLoS ONE 3(2): e1551. doi:10.1371/journal.pone.0001551

Editor: Holger Bruggemann, Max Planck Institute for Infection Biology, Germany

Received October 24, 2007; Accepted January 2, 2008; Published February 6, 2008

Copyright: (c) 2008 Nakatsuji et al. This is an open-access article distributed under the terms of the Creative Commons Attribution License, which permits unrestricted use, distribution, and reproduction in any medium, provided the original author and source are credited.

Funding: This work was supported by National Institutes of Health Grants [R01-AI067395-01, R21-R022754-01, R21-I58002-01 (C-MH) and P30-Al36214-12S1 (Y$\mathrm{TL})]$.

Competing Interests: The authors have declared that no competing interests exist.

*E-mail: chunming@ucsd.edu

\section{Introduction}

$P$. acnes, a gram-positive bacterium, is strongly associated with acne vulgaris. Isotretinoin, 13-cis-retinoic acid, is a vitamin Aderived retinoid that has been widely prescribed for systemic treatment of severe acne. However, the teratogenicity of isotretinoin is well documented [1,2]. Although isotretinoin was first approved in the United State in 1982 for treating severe acne, its use has become tightly regulated and it is not appropriate for most acne patients. Other therapies, such as systemic antibiotic treatments, also have limitations. These therapies may kill skin bacteria nonspecifically, impacting the homeostasis of resident dermal microflora [3,4]. In addition, oral antibiotics contain the risk of harming the intestinal microflora. The presence of $P$. acnes prevents colonization by more harmful bacteria [Staphylococcus aureus (S. aureus) and Acinetobacter baumannii, and it has been reported that $P$. acnes can transfer anti-bacterial resistance to other bacteria within the resident skin microflora when systemic antibiotic therapy is used [3]. Recently, antibiotic-resistant $P$. acnes has been found with the use of antibiotics [5]. Currently available topical treatments for acne lesions, including drugs, are palliative, requiring a sustained treatment regiment. When these therapies are discontinued, acne inevitably recurs. Acne vulgaris is a multi-factorial disease associated with $P$. acnes infection, hormone regulation and immune responses $[1,3]$. The inflammatory stage of acne vulgaris is usually the greatest concern to patients, as the lesions produced may lead to scarring and adverse psychological effects. Therefore, vaccines that suppress $P$. acnes-induced inflammation and pathogenesis, while minimizing the risk of altering the homeostasis of hormones and microflora, could be clinically valuable.

The complete genome of $P$. acnes has been sequenced [6]. Genomic data has revealed many gene encoded virulence factors, including sialidase, that are involved in degrading host tissue and inducing inflammation [7]. These virulence factors, which are either secreted from $P$. acnes or anchored in its cell wall, stimulate adjacent sebocytes and keratinocytes, triggering acne inflammation. Sialidases of $P$. acnes can cleave sialoglycoconjugates to obtain sialic acids for use as carbon and energy sources [6]. Sialidase has been used previously as a vaccine target for several diseases, including influenza and bacterial pneumonia [8,9]. Thus, we chose a $P$. acnes surface sialidase (accession number: gi| 50843035) containing an LPXTG cell wall-anchoring motif in the G-terminal domain $[6,7]$ as a target for acne vaccine development. Our data demonstrated that sialidase-immunized mice demonstrated decreased $P$. acnes-induced ear swelling and reduced production of the pro-inflammatory cytokine MIP-2, providing a rational design 
of acne vaccines that may offer a new treatment for acne vulgaris and other $P$. acnes-associated diseases.

\section{Methods}

\section{Culture of $P$. acnes}

P. acnes (ATCC ${ }^{\circledR}$ 6919) was cultured on Brucella broth agar, supplemented with $5 \%(\mathrm{v} / \mathrm{v})$ defibrinated sheep blood, vitamin $\mathrm{K}$, and hemin, under anaerobic conditions using Gas-Pak (BD Biosciences, San Jose, CA) at $37^{\circ} \mathrm{C}$. A single colony was inoculated in Reinforced Clostridium Medium (Oxford, Hampshire, England) and cultured at $37^{\circ} \mathrm{C}$ until logarithmic growth phase. Bacterial pellets were harvested by centrifugation at 5,000 $\mathrm{g}$ for $10 \mathrm{~min}$.

\section{Molecular Cloning and Expression of Recombinant Sialidase}

A polymerase chain reaction (PCR) product encoding a putative mature $P$. acnes cell wall anchored sialidase (accession number: gi|50843035) was generated using gene-specific primers designed based on the $P$. acnes complete genome sequence. The forward PGR primer (5' - TAAGGCGTCTGTCGACTCAGGGAGGGCTCGGGCCGCAGATGC-3') included 16 nucleotides containing a Sal I site (GTCGAC) to match the end of the In-Fusion Ready pEcoli-Nterm 6xHN vector (Clontech Laboratories, Inc., Mountain View, CA), and 26 nucleotides encoding the N-terminal of sialidase. The reverse PGR primer (5'- CAGAATTCGCAAGGTTGTCTCGTGTGTGGGGCAAACTAG-3') consisted of 16 nucleotides containing a Hind III site (AAGCTT) to match the end of the vector and 23 nucleotides encoding the C-terminal of the protein. PCR was performed using the forward and reverse primers and $P$. acnes genomic DNA as template. The amplified fragment was inserted into an In-Fusion Ready pEcoliNterm expression plasmid. Competent cells (E. coli, BL21 (DE3), Invitrogen, Carlsbad, CA) were transformed with this plasmid, selected on Luria-Bertani (LB)-plates containing ampicillin $(50 \mu \mathrm{g} / \mathrm{ml})$ and an isolated colony was cultured overnight at $37^{\circ} \mathrm{C}$ with gentle shaking. An aliquot of the overnight culture was diluted 1:20 with LB-medium and incubated at $37^{\circ} \mathrm{C}$ until reaching $\quad \mathrm{OD}_{600}=0.7$. Isopropyl- $\beta$-D-thiogalactoside (IPTG) $(1 \mathrm{mM})$ was added into culture for $4 \mathrm{~h}$ to induce protein synthesis. Bacteria were harvested by centrifugation, rinsed with phosphate buffered saline (PBS), and suspended in 1/10 volume PBS. The bacteria were disrupted by sonication on ice for $1 \mathrm{~min}$ and lysed by centrifuging at 3,000 $\mathrm{g}$ for $30 \mathrm{~min}$. The pellet was washed with PBS and dissolved in $50 \mathrm{mM}$ sodium phosphate buffer containing $6 \mathrm{M}$ guanidine $\mathrm{HCl}$ and $300 \mathrm{mM} \mathrm{NaCl}$. The expressed protein possessing $6 \mathrm{x} \mathrm{HN}$ tag was purified in denaturing condition with a TALON Express Purification Kit (Clontech Laboratories, Inc., Mountain View, CA). Subsequently, the purified protein was dialyzed against $\mathrm{H}_{2} \mathrm{O}$, and then lyophilized. The lyophilized protein was dissolved in ethylene glycol $(1 \mathrm{mg} / 1.2 \mathrm{ml})$, and then stirred in a refolding buffer $(10 \mathrm{ml}, 250 \mathrm{mM}$ Tris-HCl buffer, $\mathrm{pH}$ 8.4, containing $5 \mathrm{mM}$ cysteine, $0.5 \mathrm{mM}$ cystine, and $1.5 \mathrm{M}$ urea) at $4{ }^{\circ} \mathrm{C}$ overnight. The refolded protein was dialyzed against PBS (pH 6.0), and concentrated. 10\% SDS-polyacrylamide gel electrophoresis (SDS-PAGE) and subsequent gel staining with Coomassie blue were used for detection of protein expression.

\section{Protein Identification via NanoLC- LTQ MS/MS Analysis}

In-gel digestion with trypsin and protein identification via NanoLG-LTQ mass spectrometry (MS) analysis were performed essentially as described previously [10]. The automated NanoLCLTQMS/MS setup consisted of an Eksigent Nano 2D LG system, a switch valve, a C18 trap column (Agilent, Santa Clara, CA), and a capillary reversed phased column $(10 \mathrm{~cm}$ in length, $75 \mu \mathrm{m}$ i.d. $)$ packed with $5 \mu \mathrm{m}, \mathrm{C} 18$ AQUASIL resin with an integral spray tip (Picofrit, $15 \mu \mathrm{m}$ tip, New Objective, Woburn, MA). A reversedphase LG directly coupled to a LTQ ion trap mass spectrometer (Thermo Electron, Waltham, MA) was run using a linear gradient elution from buffer $\mathrm{A}\left(\mathrm{H}_{2} \mathrm{O}\right.$ plus $0.1 \%$ formic acid $)$ to $50 \%$ buffer A plus $50 \%$ buffer $\mathrm{B}$ (acetonitrile plus $0.1 \%$ formic acid) in $100 \mathrm{~min}$. The instruments were operated in the data dependent mode. Data on the four strongest ions above an intensity of $2 \times 10^{5}$ were collected with dynamic exclusion enabled and the collision energy set at 35\%. Large-scale MS/MS spectra were extracted using default value by Bioworks ${ }^{\circledR} 3.2$ (Thermo Scientific, San Jose, CA). Charge state deconvolution and deisotoping were not performed. All MS/MS spectra were analyzed using in-house Sorcerer ${ }^{\mathrm{TM}} 2$ system with SEQUEST (v.27, rev. 11) as the search program for protein identification. SEQUEST was set up to search the target-decoy ipi.MOUSE.v3.14 database containing protein sequences in both forward and reverse orientations (68627 entries in each orientation) using trypsin as the digestion enzyme with the allowance of up to five missed cleavages. The false positive rates were roughly determined by doubling the ratio between the number of decoy hits and the total number of hits. SEQUEST was searched with a fragment ion mass tolerance of $0.5 \mathrm{Da}$ and a parent ion tolerance of $1.0 \mathrm{Da}$.

\section{Sebocyte Culture and Cell Death Determination}

An immortalized human sebocyte line SZ95 was obtained as a gift from Dr. Zouboulis CG in the Departments of Dermatology and Immunology, Dessau Medical Center, Dessau, Germany. Sebocytes were cultured in Sebomed Basal medium (Biochrom, Berlin, Germany), supplemented with $5 \mathrm{ng} / \mathrm{ml}$ human recombinant epidermal growth factor (Sigma, St. Louis, MO), 10\% (v/v) heat-inactivated fetal bovine serum, at $37^{\circ} \mathrm{C}$ under atmosphere of $5 \%(\mathrm{v} / \mathrm{v}) \mathrm{CO}_{2}$ in air. For determination of sialidase activity and the effect of sialidase on the sebocytes' susceptibility to $P$. acnes infection, sebocytes $\left(1.5 \times 10^{5}\right)$ were incubated in a 96 -well micro plate with $10 \mu \mathrm{g} / \mathrm{ml}$ of recombinant sialidase or green fluorescent protein $(\mathrm{GFP})$ in the medium with the $\mathrm{pH}$ adjusted to 6.0 for $2 \mathrm{~h}$. Incubation with the same volume of PBS served as a control. The sebocytes treated with sialidase or GFP were then co-cultured with $P$. acnes [multiplicity of infection (MOI) 1:10/cell: bacteria] for $18 \mathrm{~h}$. After the co-culture, unbound bacteria were extensively washed three times with PBS. Dead sebocytes stained with trypan blue were counted on a hemocytometer. The colony forming unit (CFU) of $P$. acnes incorporated with sebocytes was determined by spreading serial dilutions of aliquots of trypsinized sebocyte suspension in $0.01 \%(\mathrm{w} / \mathrm{v})$ Triton-X on agar plates to quantify CFU/cell. The adherence of $P$. acnes to sebocytes was visualized by staining with Accustain Gram stain kit (Sigma, St. Louis, MO).

\section{Flow Cytometry}

Sebocytes $\left(1.5 \times 10^{6}\right)$ were incubated with recombinant sialidase $(10 \mu \mathrm{g} / \mathrm{ml})$ at $\mathrm{pH} 6.0$ for $2 \mathrm{~h}$. The sebocytes were washed with PBS three times and fixed with 1\% formaldehyde in PBS for $5 \mathrm{~min}$ at room temperature. After washing, the cells were incubated at $37^{\circ} \mathrm{C}$ for $15 \mathrm{~min}$ with $10 \mu \mathrm{g} / \mathrm{ml}$ of biotinylated MAA lectin I (Vector Laboratories, Burlingame, CA), which was prepared with $1 \%(\mathrm{w} / \mathrm{v})$ bovine serum albumin (BSA) in PBS. The bound biotin was reacted with $1.5 \mu \mathrm{g} / \mathrm{ml}$ of a streptavidin-streptavidin-fluorescein isothiocyanate (FITG) conjugate (Jackson immunoresearch, West Grove, PA), which was incubated in $1 \%(\mathrm{w} / \mathrm{v})$ BSA in PBS at $37^{\circ} \mathrm{C}$ for $10 \mathrm{~min}$. After trypsinizing, the fluorescence intensities of the cells were analyzed with a flow cytometer (FACSCalibur, BD Biosciences, San Jose, CA). 


\section{Vaccination and Antibody Detection}

Female ICR mice approximately 3-months-old (Harlan, Indianapolis, IN) were used for vaccination. Recombinant sialidase or GFP was dissolved in PBS and mixed with an equal volume of complete or incomplete Freund's adjuvant. For the first vaccination, $50 \mu \mathrm{g}$ of recombinant protein in complete Freund's adjuvant was injected subcutaneously into the dorsal skin. Two weeks later, $50 \mu \mathrm{g}$ of recombinant protein in incomplete Freund's adjuvant was intraperitoneally injected for second boost. One week after the second boot, serum containing immunoglobulin $G$ ( $\operatorname{IgG})$ antibody was harvested for western blot analysis. Serum was diluted 1:10,000 for the reaction. To obtain antiserum against $P$. acnes, ICR mice were vaccinated intranasally with killed $P$. acnes $\left(25 \mu \mathrm{l} ; 10^{8} \mathrm{CFU}\right)$ for nine weeks (three boosts at three-week intervals). $P$. acnes was killed by heat at $65^{\circ} \mathrm{C}$ for $30 \mathrm{~min}$ or ultraviolet (UV) at 7,000 mJ/ $\mathrm{cm}^{2}$. Anti-serum raised against killed $P$. acnes was collected one week after the third boost. Each group $(\mathrm{n}=4)$ and each experiment was performed in triplicate. $10 \%$ SDS-PAGE was used for western blot analysis. All experiments using mice were conducted according to institutional guidelines.

\section{Immune Protection by A Sialidase-based Vaccine}

Live $P$. acnes ( $\left.20 \mu \mathrm{l} ; 10^{7} \mathrm{CFU}\right)$ was subcutaneously injected in the central portion of the left ear of sialidase- or GFP- vaccinated mice. $20 \mu \mathrm{l}$ of PBS was injected into the right ear as a control. After injection, ear thickness was measured using a micro caliper (Mitutoyo, Aurora, IL) and recorded periodically until ear swelling had nearly subsided (71 days). The $P$. acnes-induced change in ear thickness was calculated as \% of that in PBS-injected ears. To assess the effect of vaccination on $P$. acnes growth, ears injected with PBS or live $P$. acnes in sialidase- or GFP-immunized mice were excised and homogenized at eight days after injection. $P$. acnes from homogenized ears were grown on agar plates for CFU counting.

\section{Tissue Chamber and Pro-inflammatory Cytokine Detection}

ICR mice were anesthetized with $10 \mathrm{mg}$ of ketamine and $1.5 \mathrm{mg}$ of xylazine per $100 \mathrm{~g}$ of body weight. The tissue chamber (internal and external diameters, 1.5 and $3.0 \mathrm{~mm}$, respectively) consisted of closed ploytetrafluoroethylene Teflon cylinders with 12 regularly spaced $0.1 \mathrm{~mm}$ holes. The tissue chamber was sterilized by soaking in $70 \%$ ethanol overnight. The sterile tissue chamber was then implanted subcutaneously under abdominal skin and maintained in the mice for 7 days to ensure the chamber was fully integrated with the subcutaneous environment. For histological observation, an implanted tissue chamber was cross-sectioned, stained with haematoxylin and eosin (H\&E) (Sigma diagnostics, St. Louis, MO), and viewed on a Zeiss Axioskop2 plus microscope (Carl Zeiss, Thornwood, NY). Fluids in the tissue chamber were drawn by pecutaneous aspiration before and after $P$. acnes $\left(20 \mu \mathrm{l} ; 10^{7} \mathrm{CFU}\right)$ injection for three days. The concentrations of MIP-2 and TNF- $\alpha$ in the tissue chamber fluid were determined with sandwich enzymelinked immunosorbent assay (ELISA) kits (R \& D Systems Inc., Minneapolis, $\mathrm{MN}$ ) according to the provided protocols.

\section{In Vitro Neutralization Assays}

For in vitro neutralization assays, $P$. acnes $\left(2 \times 10^{6} \mathrm{CFU}\right)$ was pretreated with $2.5 \%(\mathrm{v} / \mathrm{v})$ of anti-sialidase or -GFP serum in the medium at $37^{\circ} \mathrm{C}$ for $2 \mathrm{~h}$ before being added to sebocyte $\left(2 \times 10^{5}\right)$ cultures for $18 \mathrm{~h}$. Complement in the serum was deactivated by heating at $56^{\circ} \mathrm{C}$ for $30 \mathrm{~min}$. Sebocyte death induced by $P$. acnes was determined with $p$-Nitrophenyl phosphate disodium ( $p$ NPP) (Pierce, Rockford, IL) according to the method of Martin and Clynes [11].
The sebocytes were washed with PBS three times and reacted with $p$ NPP for $1 \mathrm{~h}$ at $37^{\circ} \mathrm{C}$. The absorbance of the $p$ NPP reaction was measured at $\mathrm{OD}_{405} \mathrm{~nm}$. As a baseline, sebocytes killed with $0.1 \%$ (v/ v) Triton-X were also assayed. Sebocyte death induced by $P$. acnes cytotoxicity was calculated as (the $\mathrm{OD}_{405}$ difference without and with $P$. acnes treatment $) /\left(\right.$ the $\mathrm{OD}_{405}$ difference without $P$. acnes and with Triton-X treatment $) \times 100 \%$. For IL-8 detection, $P$. acnes $\left(1.5 \times 10^{8}\right.$ CFU) pre-treated with anti-sialidase or anti-GFP sera was added into sebocyte $\left(3 \times 10^{6}\right)$ cultures for $8 \mathrm{~h}$. After centrifuging to remove bacteria, the concentrations of IL- 8 in the culture medium were determined by ELISA assays (R \& D Systems Inc.).

\section{Statistical Analyses}

Data are presented as mean \pm standard error (SE). The Student $t$-test was used to assess the significance of independent experiments. The criterion $p<0.05$ was used to determine statistical significance.

\section{Results}

\section{Sialidase Expression and Mass Spectrometric Identity}

A gene encoding sialidase was amplified by PCR using specific primers from template DNA prepared from $P$. acnes. The PGR products were inserted into a pEcoli-Nterm 6xHN plasmid and expressed in E. coli [E. coli BL21 (DE3)]. After IPTG induction, overexpressed sialidase-6xNH fusion protein from $E$. coli was detected by SDS-PAGE with Coomassie blue staining at approximately $53.1 \mathrm{kDa}$ molecular weight (Figure 1A). The sialidase (Figure 1B) was purified using a TALON resin column. The sialidase expression was confirmed by NanoLC-LTQ MS/MS mass spectrometric sequencing after in-gel trypsin digestion (Figure 1C). Nineteen internal peptides (data not shown) derived from sialidase were fully sequenced by NanoLC-LTQMS/MS mass spectrometry, matching well with those from $P$. acnes sialidase (accession number: gi|50843035). An internal peptide (VVELSDGTLMLNSR; 316329 amino acid residues) of sialidase is presented (Figure 1C), validating the expression of recombinant sialidase.

\section{The Susceptibility of Sebocytes to $P$. acnes after Sialidase Treatments}

Real-time PCR revealed that the $P$. acnes expressed sialidase (Text $\mathrm{S} 1$ and Figure $\mathrm{S} 1$ ). The experiment was conducted by using a triacylglycerol lipase of $P$. acnes as a positive control. To determine its enzymatic activity, purified sialidase $(10 \mu \mathrm{g} / \mathrm{ml})$ was added to human SZ95 sebocyte cultures for $2 \mathrm{~h}$ to remove the sialic acids on the surface of sebocytes. Surface sialic acids were quantified by flow cytometry (FACSCalibur, BD Biosciences, San Jose, CA) using biotinylated Maackia Amurensis (MAA) lectin I and FITG conjugate. The fluorescence of MAA labeled-sialic acids in sialidase-treated sebocytes was reduced by approximately 69\% (Figure 2A, a), whereas the fluorescence in control sebocytes treated with GFP was unchanged (Figure 2A, b). These data indicate that our purified recombinant sialidase enzymatically cleaves sialoglycoconjugates, releasing sialic acids. Treatment of purified sialidase for $2 \mathrm{~h}$ did not influence the cell viability of sebocytes (data not shown). After treatment with sialidase, or controls, for $2 \mathrm{~h}$, sebocytes $\left(5 \times 10^{6}\right.$ cells $)$ were exposed to live $P$. acnes $\left(5 \times 10^{7} \mathrm{CUF}\right)$ overnight. Live $P$. acnes induced approximately $15 \sim 20 \%$ cell death in PBS (vehicle)- or GFP-treated sebocytes, whereas sialidase-treated sebocyte cell death was significantly higher, at $33.5 \pm 1.8 \%$ (Figure $2 \mathrm{~B}$ ), suggesting that sialidase treatment increases the susceptibility of sebocytes to $P$. acnes. It has been demonstrated that incubation of human buccal epithelial cells with sialidase greatly augments Pseudomonas aeruginosa adherence [12], leading us to examine the adherence of $P$. acnes to sialidase- 
A

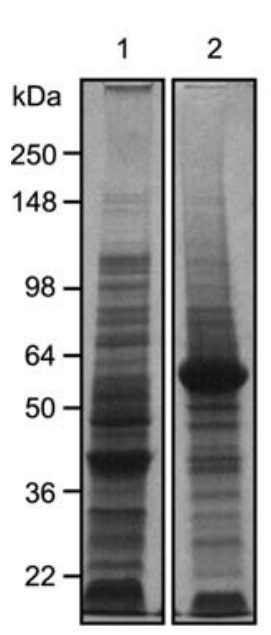

B

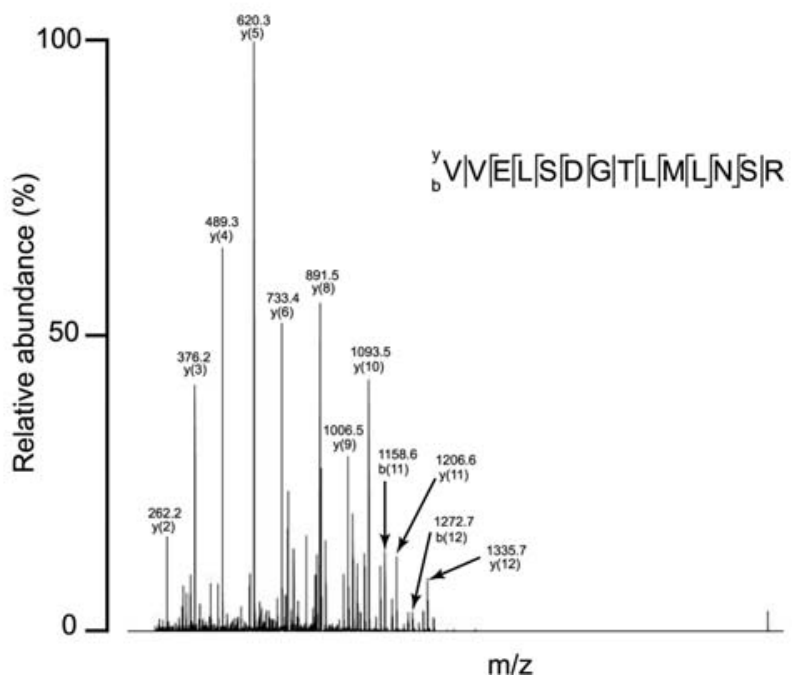

Figure 1. Expression and Purification of A Cell Wall Anchored Sialidase. (A) A vector encoding a cell wall anchored sialidase (accession \# gi|50843035) was constructed by inserting a PCR amplified full sialidase gene into the pEcoli-Nterm 6xHN vector (Clontech Laboratories, Inc., Mountain View, CA) at the Sall and Hindlll restriction sites. Specific primers including the sense (5'- ATGACTTTGACCACGAAACTGAGCG-3') and antisense primers (5'-TCAGGCAGGGCTCCGGCCCCAGATGC-3') were designed to clone sialidase from P. acnes (ATCC 6919). The vector, which contains $\mathrm{T} 7 / \mathrm{LacO}$ promoter, is derived from the pET system developed by William Studier and colleagues to achieve exceptionally high levels of protein expression in E. coli. Sialidase (arrow) was expressed in E. coli in the absence (lane 1) or presence (lane 2) of (1 mM) IPTG. After IPTG induction, sialidase was successfully expressed in E. coli and shown at about $53.1 \mathrm{kDa}$ on a $10 \%$ SDS-PAGE (arrowhead). (B) Purified sialidase (arrow) was obtained via In-Fusion Ready TALON Express Bacterial Expression and Purification kit (Clontech Laboratories, Inc., Mountain View, CA). (C) The expression and purity of sialidase were confirmed by Nano-LTQ MS/MS mass spectrometry (Thermo Electron Corp. Waltham, MA). A sequenced internal peptide (VVELSDTLMLNSR) of sialidase was presented.

doi:10.1371/journal.pone.0001551.g001

treated sebocytes. Pre-treatment with sialidase, but not GFP $(10 \mu \mathrm{g} /$ $\mathrm{ml}$ for $2 \mathrm{~h}$ ), significantly increased the association of $P$. acnes with sebocytes (Figure 2, D). Accustain Gram stains also clearly indicated that the number of $P$. acnes interacting with sebocytes was elevated once sebocyte surface sialic acids were removed (Figure 2D, a-c). These data, combined with the fact that sialidase is a surface protein carrying a cell wall-anchoring LPXTG motif, make it a potentially valuable target for creating vaccines against $P$. acnes-associated diseases, such as acne vulgaris.

\section{Immunogenicity in Mice Vaccinated with Recombinant Sialidase}

To assess the immunogenicity of sialidase, ICR mice were vaccinated with heat killed $P$. acnes for nine weeks. Recombinant sialidase, GFP and $P$. acnes lysates were subjected to western blot analysis. Many proteins with molecular weights greater than $50 \mathrm{kDa}$ were immunoreactive with mouse serum obtained from the heat killed $P$. acnes-immunized mice (Figure 3A, lane 3), however, sialidase was not (Figure 3A, lane 1), indicating that mice immunized with whole organism $P$. acnes do not produce antibodies to sialidase. Similarly, recombinant sialidase was not immunoreactive to mouse serum obtained from UV-killed $P$. acnes-immunized mice (Figure S2), indicating that the undetected immunogenicity was not due to denaturation of sialidase during the heat treatment. We next vaccinated ICR mice with recombinant sialidase, or a GFP control, using Freund/(in)complete adjuvants. Antibody production in the serum of immunized mice was detected by western blot analysis four weeks after immunization (Figure 3B, lane 1). A strong band appearing at $53.1 \mathrm{kDa}$ was visualized when purified sialidase was reacted with serum from sialidase-immunized mice, indicating that sialidase was immunogenic in mice vaccinated with recombinant sialidase. No immunoreactivity against sialidase was detectable in GFP-immunized mice (Figure 3B, lane 2).

\section{Protective Immunity Against $P$. acnes Challenge in Sialidase-vaccinated Mice}

To asses immune protection in vivo, ICR mice immunized with recombinant proteins (sialidase or GFP) along with Freund/ (in)complete adjuvants were challenged with live $P$. acnes $\left(10^{7}\right.$ $\mathrm{CFU})$ three weeks after vaccination. One ear of each mouse was subcutaneously injected with $25 \mu \mathrm{l}$ of $P$. acnes $\left(10^{7} \mathrm{CFU}\right)$ and the other ear was injected with $25 \mu \mathrm{l}$ of PBS as a control. Injection of $P$. acnes induced ear swelling (Figure 4A) and redness (Figure 4B). Ear thickness was measured regularly for 71 days, revealing a biphasic ear-swelling pattern. Ear thickness in GFP-immunized mice rapidly increased more than two fold $(215.8 \pm 7.7 \%) 32 \mathrm{~h}$ after $P$. acnes challenge, decreased, then rebounded four days after challenge. Ear swelling was significantly reduced in both phases (at 32 hours and 4 days post-challenge) by more than $50 \%$ when mice were immunized with sialidase (Figure 4A). Sialidase immunization also resulted in decreased erythema in ears challenged with $P$. acnes (Figure 4B). Ear swelling in GFPimmunized mice nearly subsided 71 days after $P$. acnes challenge, whereas sialidase-immunized mice were completely recovered 58 days after challenge. These results indicate that sialidaseimmunized mice suppressed $P$. acnes-induced ear inflammation.

\section{Detection of Pro-inflammatory Cytokines in Implanted Tissue Chambers}

Induction of pro-inflammatory cytokines also plays a key role in the progression of acne vulgaris. To determine whether sialidase immunization alters the level of $P$. acnes-induced pro-inflammatory cytokines, we employed a tissue chamber model (Figure 5A) to collect pro-inflammatory cytokines in vivo. The tissue chamber model has been extensively characterized in mice $[13,14]$ and accurately mimics bacterial infections in vivo. A tissue chamber was implanted subcutaneously in the abdomen of ICR mice 7 days 
A

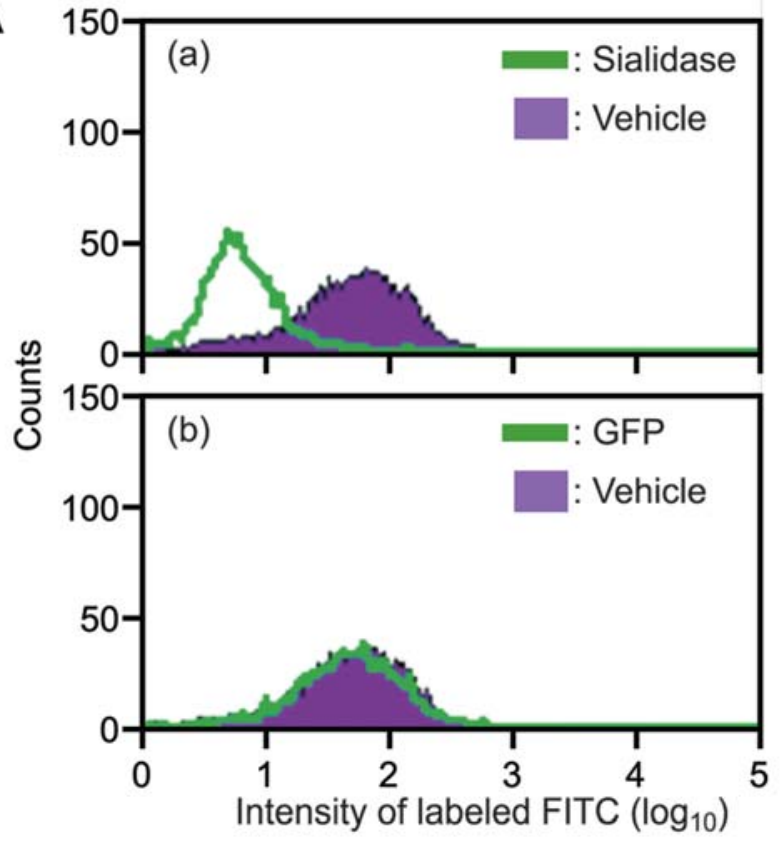

B

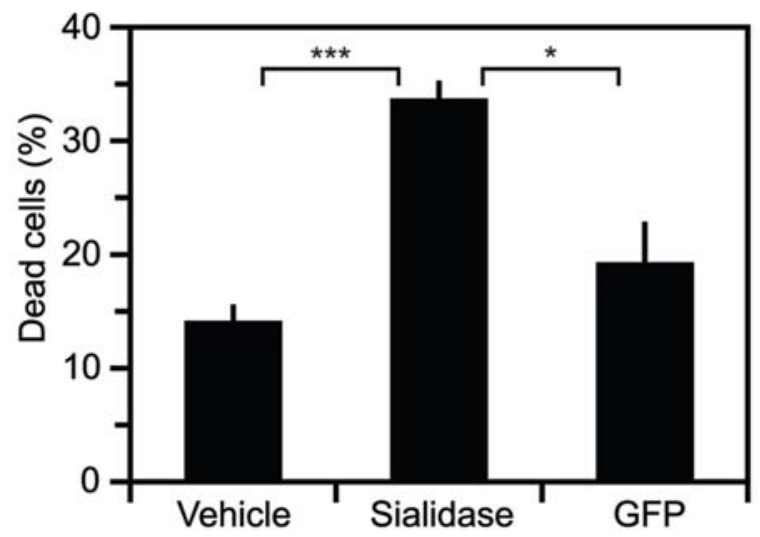

C

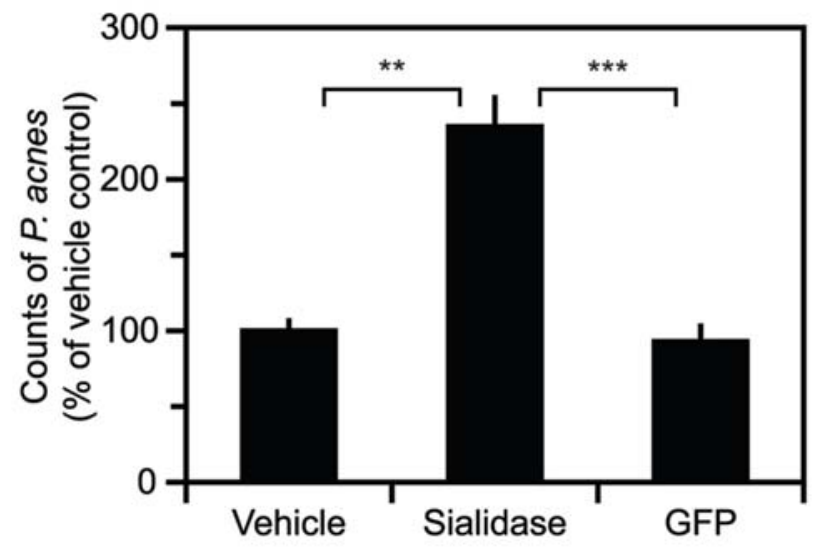

D

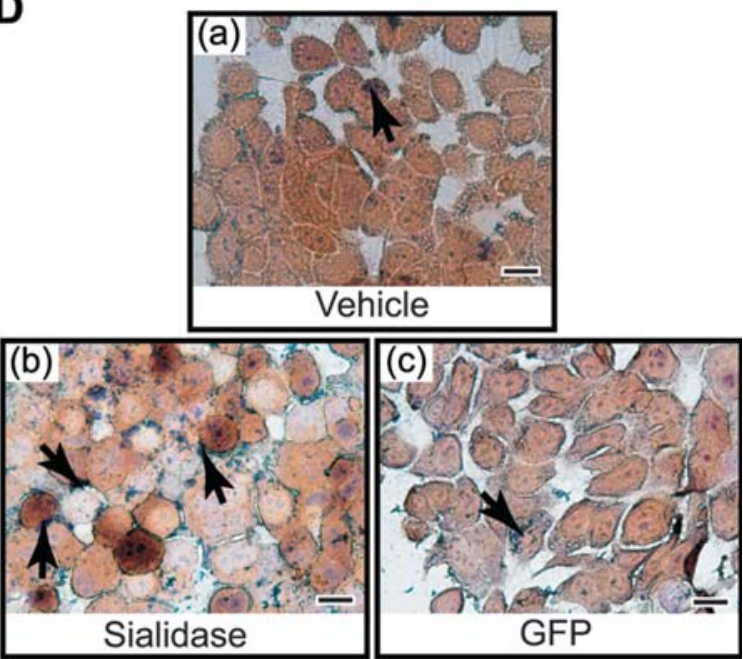

Figure 2. Removal of Sialic Acids from Human Sebocytes by Sialidase Increases Their Susceptibility to $P$. acnes. (A) Sialic acids on the cell surface of immortalized human sebocytes (SZ95) were detected by their reaction with biotinylated MAA lectin I (10 $\mu \mathrm{g} / \mathrm{ml})$ and streptavidin-FITC conjugate. FITC-fluorescence intensity was measured by flow cytomtery (FACSCalibur, BD Biosciences, San Jose, CA), reflecting the quantity of sialic acid. The sebocytes were pre-treated with $10 \mu \mathrm{g} / \mathrm{ml}$ of purified recombinant sialidase (green, a), GFP (green, b) or an equal volume of PBS (vehicle) (purple) at $\mathrm{pH} 6$ for $2 \mathrm{~h}$. The decrease in FITC-fluorescence intensity in sialidase-treated sebocytes validated the enzymatic activity of the purified sialidase. (B) After pre-treatment with sialidase, sebocytes were co-cultured with P. acnes $\left(5 \times 10^{7} \mathrm{CFU} / 5 \times 10^{6}\right.$ cells) for $18 \mathrm{~h}$. $P$. acnes-induced cell death in PBS (vehicle)-, sialidase- or GFP-pretreated sebocytes was assessed by trypan blue. Cell death is presented as the \% of dead cells compared to all cultured cells. (C) After washing out unbound P. acnes, the number of $P$. acnes adhered to sebocytes was calculated by spreading Triton-X $(0.01 \%)$ lysed sebocytes on agar plates to quantify CFU/cell. (D) The CFU/cell in vehicle-pretreated sebocytes was defined as $100 \%$. Adherence of $P$. acnes (arrows) to vehicle (a)-, sialidase (b)- or GFP (c)-treated sebocytes was visualized by staining with the Accustain Gram stain kit. Pre-treatment with sialidase significantly increased the adhesion of $P$. acnes to sebocytes. Shown are representative results of three independent experiments. Error bars are mean \pm SE $\left(P<0.01^{*}, P<0.001^{* *}, P<0.0005^{* * *}\right.$ by Student's t-test $)$. Bar: $10 \mu \mathrm{m}$.

doi:10.1371/journal.pone.0001551.g002

before $P$. acnes $\left(10^{7} \mathrm{CFU}\right)$ inoculation. Three days after $P$. acnes inoculation, tissue chamber fluids containing pro-inflammatory cytokines were drawn by percutaneous aspiration and the levels of MIP-2 (Figure 5B) and (tumor necrosis factor) TNF- $\alpha$ were measured by ELISA. In GFP-immunized mice, a significant increase in the level of MIP-2 was observed 3 days after $P$. acnes inoculation, while sialidase-immunized mice demonstrated $61 \%$ less induction. The level of TNF- $\alpha$ remained unchanged after $P$. acnes inoculation in both GFP- and sialidase-immunized mice (data not shown). These results suggest that a vaccine targeting sialidase effectively suppresses $P$. acnes-induced production of the proinflammatory cytokine MIP-2 in the mice. 
A

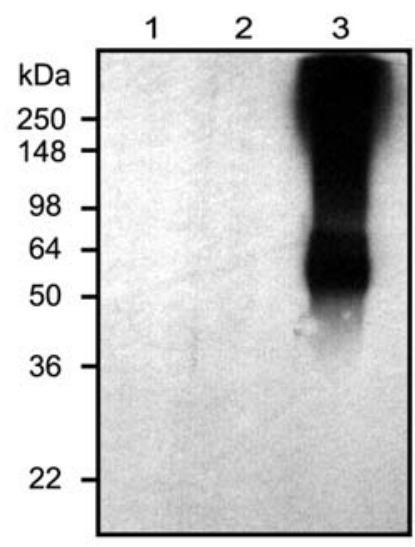

B

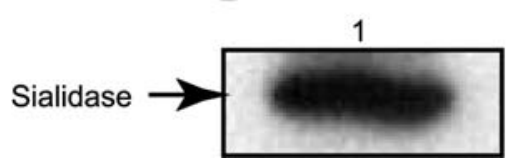

Figure 3. Mice Immunized with Recombinant Sialidase and Freund (in)complete Adjuvants Produce Sialidase Specific Antibodies. (A) ICR mice were vaccinated with heat-killed $P$. acnes for nine weeks (three boosts at a three-week interval). Serum (1: 500 dilution), harvested one week after the third boost, was reacted to recombinant sialidase $(1 \mu \mathrm{g}$; lane 1$)$, GFP $(1 \mu \mathrm{g}$, lane 2$)$, and $P$. acnes lysates $(7 \mu \mathrm{g}$, lane 3$)$ that had been run on a $10 \%$ SDS-PAGE. The $6 \mathrm{x} \mathrm{HN}$ tag of recombinant sialidase was removed by enterokinase before loading into a SDS-PAGE. Although heat-killed $P$. acnes-vaccinated mice produced anti-sera against several $P$. acnes proteins ( $>50 \mathrm{kDa}$ ) (lane 3 ), sialidase specific antibodies were not detected (lane 3). (B) ICR mice were then vaccinated with a recombinant sialidase (lane 1) or GFP (lane 2) using Freund/(in)complete adjuvants. For the first vaccination, mice were subcutaneously inoculated with $50 \mu \mathrm{g}$ of sialidase or GFP, which were emulsified with a complete Freund adjuvant. Two weeks later, the second vaccination was delivered via intramuscular injection with the same amount of antigen mixed well with incomplete Freund adjuvant. Anti-sialidase antibodies were detected by western blot one week after the second vaccination. $1 \mu \mathrm{g}$ of recombinant sialidase or GFP were separated via $10 \%$ SDS-PAGE, transferred to a PVDF membrane and reacted with mouse sera $(10,000$-fold dilution). Sialidase antibodies were provoked when mice were immunized with recombinant sialidase, but not with GFP. Data is representative of three separate experiments with similar results.

doi:10.1371/journal.pone.0001551.g003

\section{Neutralization of Cytotoxicity of $P$. acnes to Human Sebocytes with Anti-sialidase Serum: Implication for Acne Treatment}

In order to evaluate whether anti-sialidase serum was capable of neutralizing $P$. acnes cytotoxicity for sebocytes, $P$. acnes was preincubated with anti-sialidase serum at $37^{\circ} \mathrm{C}$ for $2 \mathrm{~h}$ prior to being added to sebocyte cultures. The CFU counting on agar plates showed that $2 \mathrm{~h}$ incubation with antisera did not impair the growth of $P$. acnes (data not shown). $P$. acnes pre-incubated with anti-GFP serum caused $29.3 \pm 3.4 \%$ of the sebocytes to undergo cell death, while pre-incubation with anti-sialidase serum dramatically reduced $P$. acnes-induced sebocyte death to $4.5 \pm 1.8 \%$ (Figure 6A). This data suggests that mice immunized with sialidase produce antibody that can neutralize the cytotoxicity of $P$. acnes in vitro. It has been shown that sebocytes expressed Toll-like receptor 2 (TLR2) and secreted cytokine IL-8 once they were stimulated with $P$. acnes [15]. In addition, expression of IL-8 was detectable in skin biopsies from patients with inflammatory acne vulgaris [16]. Thus, we next examine the capability of anti-sialidase serum in neutralizing $P$. acnes-induced IL-8 production in human sebocytes. Sebocytes stimulated with $P$. acnes that was pre-incubated with anti-GFP or anti-sialidase serum released IL-8 at the amount of $2.49 \pm 0.10$ or $1.76 \pm 0.08 \mathrm{ng} / \mathrm{ml}$, respectively (Figure $6 \mathrm{~B}$ ), indicating that anti-sialidase serum effectively suppresses $P$. acnes-induced IL-8 production in human sebocytes. Sebocytes within sebaceous glands are major target cells of $P$. acnes in patients with acne vulgaris [17]. Accordingly, generation of antibody against sialidase in acne patients may counteract the cytotoxicity of $P$. acnes to sebocytes and alleviate acne development.

\section{Discussions}

It has been reported that treatment of human buccal epithelial cells with the sialidase considerably increased Pseudomonas aeruginosa adherence [18]. In addition, immunization with recombinant Streptococcus pneumoniae sialidase resulted in a significant reduction in pneumococcal colonization in the chinchilla model [19]. We demonstrated that the adherence of $P$. acnes to human sebocytes was augmented after removal of sialic acids from the cell surface. This result is in agreement with previous findings that sialidase is involved in the adhesion of pathogens to host cells $[18,20]$, and that treatment of host cells with sialidase changes their susceptibility to pathogens [19]. Adhesion process of bacteria occurs at the early stage of infection and is essential for its colonization, and in turn, colonization may be required for subsequent development of symptoms of diseases. Thus, vaccination targeting sialidase of $P$. acnes may be an efficient modality for the prevention of early infection of $P$. acnes.

Patients with acne lesions are likely to produce anti- $P$. acnes antibodies [21], however, acne lesions still recur in these patients. This suggests that patients infected with $P$. acnes may develop insufficient immunity to prevent subsequent $P$. acnes infection and acne recurrence. In this study, mice were immunized with either heat or UV killed $P$. acnes or recombinant sialidase. Our data has shown that sialidase is not immunogenic if vaccination with $P$. acnes is administered whereas sialidase becomes immunogenic when vaccination with recombinant protein is performed (Figure 3). An analysis of patients' sera by western blot assay recognized a $96 \mathrm{kDa}$ antigenic component of $P$. acnes [22]. No reports demonstrated that sialidase is antigenic in the sera of acne patients [21,22]. This result suggests that acne progression and recurrence could be effectively prevented if the antibody against sialidase of $P$. acnes can be robustly elicited in acne patients.

Inactivation of $P$. acnes has been used to create vaccines against acne vulgaris [23,24]. Acnevac or autovaccines containing killed strains of $P$. acnes and/or Staphylococci have been tested in acne and normal healthy subjects [25]. Although these killed $P$. acnes-based vaccines showed a good effect on acne patients, their effect is based on the non-specific modulation of the immune system of patients. Furthermore, It has been shown that mice immunized with killed $P$. acnes demonstrate non-specific resistance to challenge with other microbes [26]. A single intraperitoneal injection of phenol-treated $P$. acnes into mice showed non-specific resistance against subsequent lethal doses of an intraperitoneal challenge of Klebsiella pneumoniae, S. aureus, and Streptococcus pyogenes (S. pypgenes) [26]. Recently, it has been shown that animals sensitized with $P$. acnes exhibit an increased susceptibility to $E$. coli lipopolysacharride (LPS) induced sepsis and [27] and liver failure [28]. The cell wall anchored sialidase presented in this article shares low identity with other surface sialidases in other pathogens [6]. Thus, acne vaccines 
A

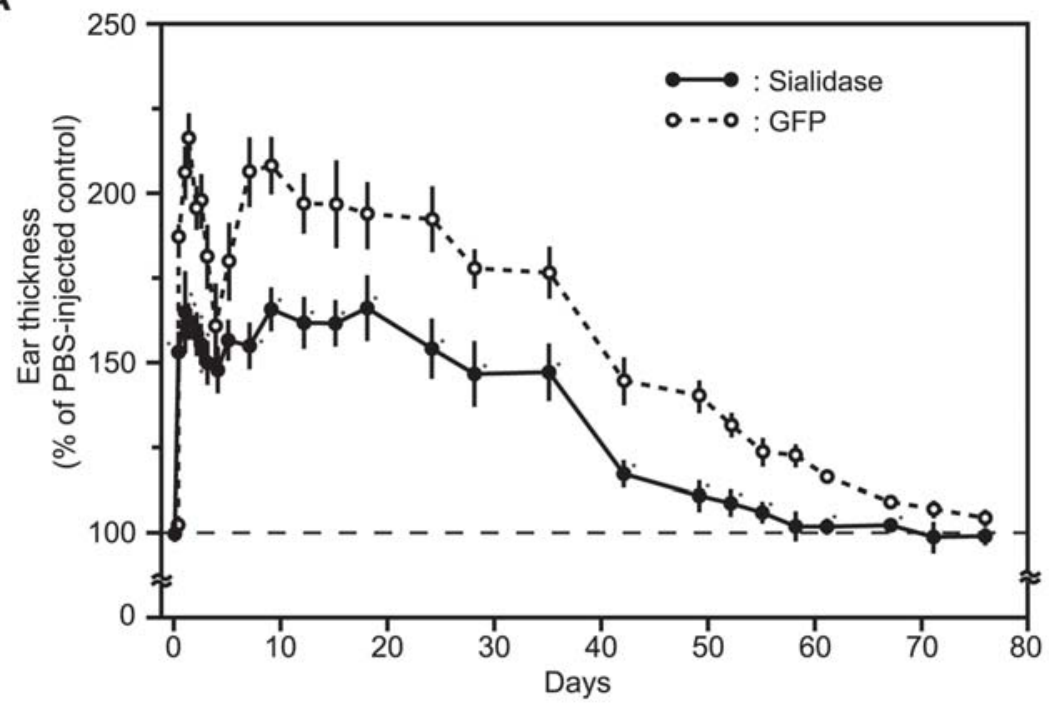

B
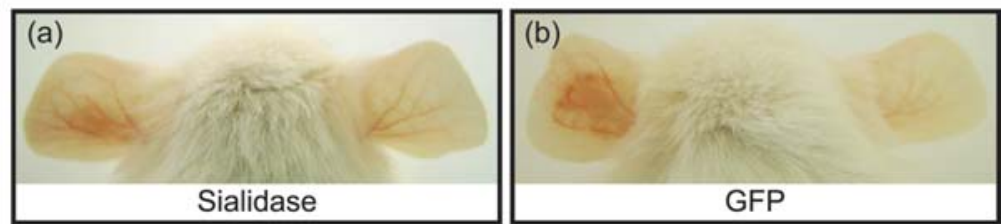

Figure 4. Immune Protection Conferred by A Sialidase-based Acne Vaccine. (A) The cytotoxicity of $P$. acnes was calculated as described in Methods and presented as mean \pm SE $\left(P<0.0005^{* *}\right.$ by Student's $t$-test). For assaying in vivo immune protection, ICR mice were immunized with recombinant sialidase or GFP using Freund (In)complete adjuvants (Figure 3). After confirming antibody production by western blot, live $P$. acnes (10 ${ }^{7}$ CFU, $25 \mu \mathrm{l}$ ) were injected subcutaneously into the ears of sialidase- and GFP-immunized mice, with PBS (25 $\mu \mathrm{l})$ as a control. Ear thickness was periodically measured for 71 days after injection and changes reported as \% of ear thickness in PBS-injected ears. $P$. acnes-induced ear swelling was significantly suppressed in sialidase-immunized mice in comparison with GFP-immunized mice $\left(P<0.05^{*}\right)$, except for day 0, 4, 5 and 71 . (B) Erythema was assessed in sialidase- (a) or GFP- (b) immunized mice $24 \mathrm{~h}$ after live $P$. acnes (left ears) or PBS (right ears) injection.

doi:10.1371/journal.pone.0001551.g004

A


B



Figure 5. A Sialidase-based Acne Vaccine Suppresses Induction of the Pro-inflammatory Cytokine MIP-2 by $P$. acnes. (A) A tissue chamber (a) (internal and external diameters, 1.5 and $3 \mathrm{~mm}$, respectively, length, $1 \mathrm{~cm}$; internal volume, $80 \mu \mathrm{l}$ ) was implanted subcutaneously in the abdomen of sialidase- or GFP-immunized mice. The tissue chamber consisted of closed ploytetrafluoroethylene Teflon cylinders with 12 regularly spaced $0.1 \mathrm{~mm}$ holes. Bar: $1 \mathrm{~cm}$. H\&E staining of the cross-section of an implanted tissue chamber showed that the chamber was completely encapsulated by fibrotic tissue 7-days after implantation (b). Bar: $1.0 \mathrm{~mm}$. P. acnes (10 $\mathrm{CFU}, 20 \mu \mathrm{l}$ ) was injected and trapped in this encapsulated tissue chamber. (B) Tissue chamber fluid containing MIP-2 was drawn by pecutaneous aspiration before (open bar) and three days after (solid bar) $P$. acnes injection. Measurement of MIP-2 was carried out by sandwich ELISA that used the Quantikine M mouse MIP-2 set (R\&D System, Minneapolis, $\mathrm{MN})$. Vaccination with sialidase markedly suppressed the $P$. acnes-induced increase in MIP-2. Error bars represented mean \pm SE of five separate experiments $(* P<0.005$ by Student's $t$-test).

doi:10.1371/journal.pone.0001551.g005 
A

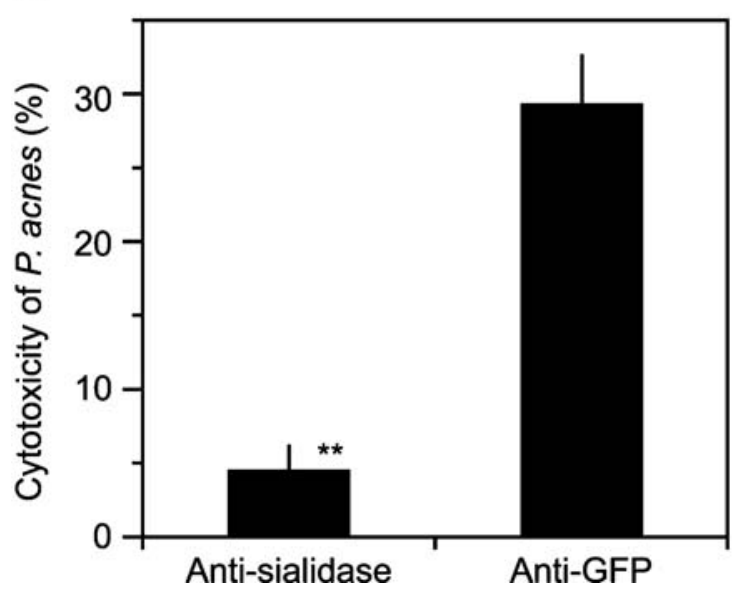

B

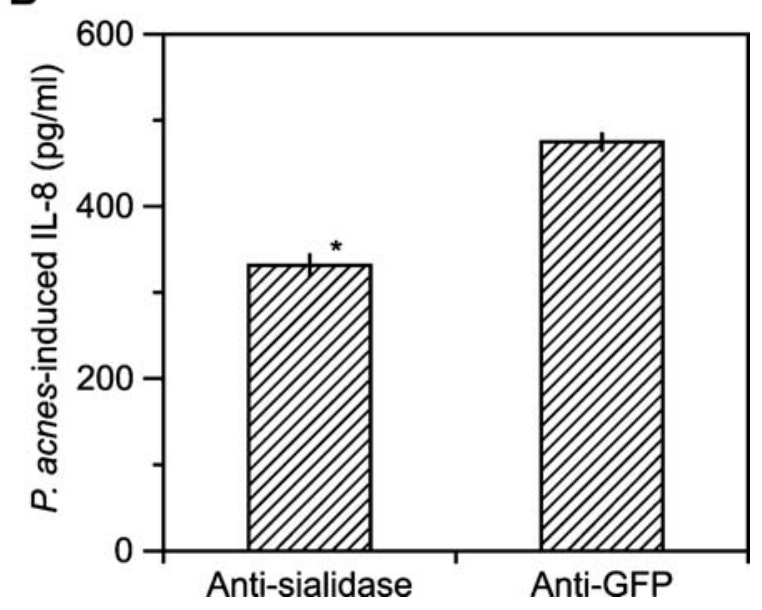

Figure 6. Neutralization with Anti-sialidase Serum Decreases $P$. acnes-induced Cell Death and IL-8 Production in Human Sebocytes. (A) For in vitro neutralization assays, P. acnes was pre-treated with $2.5 \%(\mathrm{v} / \mathrm{v})$ anti-sialidase or GFP sera at $37^{\circ} \mathrm{C}$ for $2 \mathrm{~h}$. Serum-pretreated $P$. acnes $\left(2 \times 10^{6} \mathrm{CFU}\right)$ was then co-cultured with sebocytes $\left(2 \times 10^{5}\right.$ cells) for $18 \mathrm{~h}$. Sebocyte death induced by serum-pretreated $P$. acnes was detected by $p$ NPP. The cytotoxicity of $P$. acnes was calculated as described in Methods and presented as mean $\pm \operatorname{SE}\left(n=4, P<0.0005^{* *}\right.$ by Student's $t$-test). (B) Serum-pretreated $P$. acnes $\left(1.5 \times 10^{8} \mathrm{CFU}\right)$ was co-cultured with sebocytes $\left(3 \times 10^{6}\right.$ cells) for $8 \mathrm{~h}$. Measurement of IL- 8 in the culture medium was carried out by ELISA assays using a Quantikine human IL-8 set (R\&D System). The data is presented as mean \pm SE ( $n=4, P<0.01^{*}$ by Student's $t$-test). doi:10.1371/journal.pone.0001551.g006

utilizing a $P$. acnes specific sialidase instead of killed $P$. acnes as the immunogen may be more specific and reduce the chance of side effects. There are at least five sialidases [sialidase B (gi| 50843035); cell wall anchored sialidase (gi|50843035); sialidase A precursor (gi|50842172); putative sialidase (gi|50843278) and sialidase-like protein (gi|50843043) in $P$. acnes genome. Although creation of $P$. acnes with sialidase mutation or over-expression may directly address the role of sialidase in the virulence of $P$. acnes, it may be a challenge to genetically alter all sialidases in individual $P$. acnes. However, developing a novel compound to block the enzyme activities of all sialidases in $P$. acnes may be of value [29].

Our data has demonstrated that a sialidase-based acne vaccine provided protective effects on $P$. acnes-induced ear inflammation (Figure 4). Ear thickness was measured regularly for 71 days, revealing a biphasic ear-swelling pattern. This is consistent with previous results demonstrating a biphasic change in the activity of the mouse reticuloendothelial system after intraperitoneal injection with phenol-treated $P$. acnes [26]. The biphasic pattern can be explained by two distinct stages: short-term/local (early phase) and long-term/systemic (late phase) immune stimulation. The fluctuation in the number of macrophages and other host cells reflected a biphasic pattern of $P$. acnes infection. The effect of sialidase immunization on $P$. acnes growth was also explored. Ears injected with PBS or live $P$. acnes $\left(10^{7} \mathrm{CFU}\right)$ in sialidase- or GFPimmunized mice were excised for homogenization eight days after bacterial challenge (data not shown). P. acnes from the homogenized ears were extracted and quantified on agar plates. The number of $P$. acnes in sialidase-immunized mice was not significantly different with that in GFP-immunized mice, indicating that sialidase immunization did not change the growth of $P$. acnes. Considering the data, the sialidase-based acne vaccine presented in this article may decrease $P$. acnes-induced inflammation without affecting the balance of body microflora.

Most animals including mice do not produce sufficient triglycerides in sebaceous glands to harbor $P$. acnes a fact that has encumbered the development of anti-acne vaccines and drugs targeting $P$. acnes infection [30]. Although Rhino mice with utricles that create larger follicles have been employed to determine compound comedogenicity [31], the genetic mutant mice cannot elicit antibodies against thymus-dependent antigens [32]. Thus, the use of Rhino mice as animal models may not be appropriate for vaccine evaluation. Rabbit ears have been utilized to determine compound comedogenicity of acne lesions [31]. However, the rabbit ear model has a lack of bacterial colonization and inflammation [33]. In addition, the use of rabbits may be inconvenient for vast vaccinations. A murine acne model measuring $P$. acnes-induced ear swelling and production of proinflammatory cytokines in tissue chambers may provide an alternative animal model for evaluating the potency of acne vaccines. Our data indicates that tissue chamber fluid contains various immune cells, including macrophages $\left(\mathrm{CD} 1 \mathrm{lb}^{+}\right)$, neutrophils $\left(\mathrm{Gr}^{-} \mathrm{l}^{+}\right)$, natural killer cells $\left(\mathrm{CD} 49 \mathrm{~b}^{+}\right)$and T cells $\left(\mathrm{CD}^{+}\right)$(data not shown), suggesting an influx of immune cells into an implanted tissue chamber. In addition, $\mathrm{IgG}$ against $P$. acnes was detectable in tissue chamber fluids (data not shown) when tissue chambers were implanted into heat killed $P$. acnes-immunized mice. This result indicated that antibodies are able to migrate into tissue chambers to interact with injected $P$. acnes. By growing cells in a dermisbased cell-trapped system (DBCTS) and inserting into a tissue chamber, we successfully created a tissue microenvironment in vivo [34]. Thus, a bioengineering approach using a tissue chamber integrated with DBCTS may be able to create a humanized tissue microenvironment in animals to mimic the physiological structure of human hair follicles. The approach may eventually confer an animal model for evaluating of vaccines targeting hair follicles. We detected two important murine pro-inflammatory cytokines (MIP2 and TNF- $\alpha$ ) (Figure 5). It has been reported that recognition of $P$. acnes by TLR2 induces the activation of pro-inflammatory pathways [28]. In vivo priming of mice with $P$. acnes also results in elevated serum levels of TNF- $\alpha$ [28]. The lack of elevated TNF- $\alpha$ levels in tissue chamber fluid after $P$. acnes inoculation may reflect a difference in host response between the tissue microenvironment (tissue chamber) and the systemic environment (serum).

Overall, we present a novel vaccine targeting cell wall anchored sialidase of $P$. acnes. Antibodies against sialidase provoked in vaccinated mice effectively suppressed the $P$. acnes-induced inflammation (Figures 4 and 5) and neutralized the cytotoxicity of $P$. acnes to human sebocytes (Figure 6), implicating that the sialidase-based 
vaccine may have the potential for treatment of acne vulgaris, a most common skin disease affecting $85-100 \%$ of people at some point in their lives. In addition, the sialidase-based acne vaccine may be an alternative of the killed $P$. acnes-based vaccine that performed nonspecifically and evoked many undesirable effects. Future directions include (i) establishing therapeutic acne vaccines that may benefit patients with severe acne and (ii) comparing the potency and side effects of sialidase-based vaccines with current medicines.

\section{Supporting Information}

Figure S1 Quantitative analysis of the sialidase transcript in $P$. acnes. The gene expression of sialidase was determined by real-time quantitative PCR using specific primers as described in Methods. Total RNA isolated from anaerobically cultured $P$. acnes served as a template. The gene of triacylglycerol lipase known as a pathogenic factor of $P$. acnes was used as a positive control. A pGEM-T Easy Vector (Promega, Madison, WI) inserted with PCR products was performed to estimate the number of expressed genes. The level of gene expression of sialidase and triacylglycerol lipase was normalized to that of 16SrRNA gene.

Found at: doi:10.1371/journal.pone.0001551.s001 (0.56 MB TIF)

\section{References}

1. Perry AL, Lambert PA (2006) Propionibacterium acnes. Lett Appl Microbiol 42: $185-188$.

2. Layton AM, Dreno B, Gollnick HP, Zouboulis CC (2006) A review of the European Directive for prescribing systemic isotretinoin for acne vulgaris. J Eur Acad Dermatol Venereol 20: 773-776.

3. Cooper AJ (1998) Systematic review of Propionibacterium acnes resistance to systemic antibiotics. Med J Aust 169: 259-261.

4. Ochsendorf F (2006) Systemic antibiotic therapy of acne vulgaris. J Dtsch Dermatol Ges 4: 828-841.

5. Gloor M, Wasik B, Becker A, Hoffler U (2002) Inhibition of lipase activity in antibiotic-resistant propionibacterium acnes strains. Dermatology 205: 260-264.

6. Bruggemann H, Henne A, Hoster F, Liesegang H, Wiezer A, et al. (2004) The complete genome sequence of Propionibacterium acnes, a commensal of human skin. Science 305: 671-673.

7. Bruggemann $H$ (2005) Insights in the pathogenic potential of Propionibacterium acnes from its complete genome. Semin Cutan Med Surg 24: 67-72.

8. Johansson BE, Brett IC (2007) Changing perspective on immunization against influenza. Vaccine 25: 3062-3065.

9. Tai SS (2006) Streptococcus pneumoniae protein vaccine candidates: properties, activities and animal studies. Crit Rev Microbiol 32: 139-153.

10. Martin A, Clynes M (1993) Comparison of 5 microplate colorimetric assays for in vitro cytotoxicity testing and cell proliferation assays. Cytotechnology 11: 49-58.

11. Sun B, Ranish JA, Utleg AG, White JT, Yan X, et al. (2007) Shotgun glycopeptide capture approach coupled with mass spectrometry for comprehensive glycoproteomics. Mol Cell Proteomics 6: 141-149.

12. Wolska K, Zabielska K, Jakubczak A (2006) Effect of neuraminidase on adherence of Pseudomonas aeruginosa to human buccal epithelial cells. Inhibition of adhesion by monosaccharides. Pol J Microbiol 55: 43-48.

13. Kristian SA, Lauth X, Nizet V, Goetz F, Neumeister B, et al. (2003) Alanylation of teichoic acids protects Staphylococcus aureus against Toll-like receptor 2dependent host defense in a mouse tissue cage infection model. J Infect Dis 188: $414-423$.

14. Zimmerli W, Waldvogel FA, Vaudaux P, Nydegger UE (1982) Pathogenesis of foreign body infection: description and characteristics of an animal model. J Infect Dis 146: 487-497.

15. Oeff MK, Seltmann H, Hiroi N, Nastos A, Makrantonaki E, et al. (2006) Differential regulation of Toll-like receptor and CD14 pathways by retinoids and corticosteroids in human sebocytes. Dermatology 213: 266.

16. Abd El All HS, Shoukry NS, El Maged RA, Ayada MM (2007) Immunohistochemical expression of interleukin 8 in skin biopsies from patients with inflammatory acne vulgaris. Diagn Pathol 2: 4.

17. Thiboutot D, Jabara S, McAllister JM, Sivarajah A, Gilliland K, et al. (2003) Human skin is a steroidogenic tissue: steroidogenic enzymes and cofactors are expressed in epidermis, normal sebocytes, and an immortalized sebocyte cell line (SEB-1). J Invest Dermatol 120: 905-914.

18. Kharat AS, Tomasz A (2003) Inactivation of the srtA gene affects localization of surface proteins and decreases adhesion of Streptococcus pneumoniae to human pharyngeal cells in vitro. Infect Immun 71: 2758-2765.
Figure S2 Detection of immunogenicity of sialidase in mice vaccinated with UV-killed $P$. acnes. ICR mice were vaccinated with UV-killed P. acnes as described in Methods. Serum (1: 500 dilution) was reacted to recombinant sialidase $(1 \mu \mathrm{g}$; lane 1$)$, GFP $(1 \mu \mathrm{g}$, lane 2), and $P$. acnes lysates $(7 \mu \mathrm{g}$, lane 3$)$ that had been run on a $10 \%$ SDS-PAGE. Sialidase and GFP were not immunoreactive to serum obtained from mice immunized with UV-killed $P$. acnes. Found at: doi:10.1371/journal.pone.0001551.s002 (0.33 MB TIF)

Text S1

Found at: doi:10.1371/journal.pone.0001551.s003 (0.02 MB DOC)

\section{Acknowledgments}

We thank Y. Shi for mass spectrometric analysis, D. -Y. Lee for technical advice and R. A. Dorschner and M. Kao for their critical reading of the manuscript.

\section{Author Contributions}

Conceived and designed the experiments: CMH. Analyzed the data: YL CMH TN CPH RG. Wrote the paper: CMH. Other: Designed the study: RG CMH. Collected data or performed experiments for this study: TN $\mathrm{CMH}$ CPH. Contributed to writing the papers: CMH TN.

19. Tong HH, Li D, Chen S, Long JP, DeMaria TF (2005) Immunization with recombinant Streptococcus pneumoniae neuraminidase NanA protects chinchillas against nasopharyngeal colonization. Infect Immun 73: 7775-7778.

20. Winter C, Schwegmann-Wessels C, Cavanagh D, Neumann U, Herrler G (2006) Sialic acid is a receptor determinant for infection of cells by avian Infectious bronchitis virus. J Gen Virol 87: 1209-1216.

21. Basal E, Jain A, Kaushal GP (2004) Antibody response to crude cell lysate of propionibacterium acnes and induction of pro-inflammatory cytokines in patients with acne and normal healthy subjects. J Microbiol 42: 117-125.

22. Dalen A, Hellgren L, Iversen OJ, Vincent J (1980) Antibodies against extractable components from Propionibacterium acnes in humans with and without acne vulgaris. Arch Dermatol Res 269: 253-259.

23. Zaluga E (1998) [Skin reactions to antigens of propionibacterium acnes in patients with acne vulgaris treated with autovaccine]. Ann Acad Med Stetin 44: 65-85.

24. Marcinkiewicz J, Bryniarski K, Nowak B, Dzielska D, Payerhin F (1991) [Immunomodulating properties of Acnevac vaccine]. Med Dosw Mikrobiol 43: $167-174$.

25. Loveckova Y, Havlikova I (2002) A microbiological approach to acne vulgaris. Biomed Pap Med Fac Univ Palacky Olomouc Czech Repub 146: 29-32.

26. Kobayashi F, Nagoya T, Koshi T, Saino Y (1980) Biphasic protection against bacterial infection in mice induced by vaccination of Propionibacterium acnes. Infect Immun 27: 391-396.

27. Mochizuki H, Nomura T, Kawamura I, Mitsuyama M (2005) Enhanced resistance to Gram-positive bacterium and increased susceptibility to bacterial endotoxin in mice sensitized with Propionibacterium acnes: involvement of Tolllike receptor. FEMS Immunol Med Microbiol 43: 287-293.

28. Romics LJr, Dolganiuc A, Velayudham A, Kodys K, Mandrekar P, et al. (2005) Toll-like receptor 2 mediates inflammatory cytokine induction but not sensitization for liver injury by Propioni- bacterium acnes. J Leukoc Biol 78: 1255-1264.

29. Taylor G (1996) Sialidases: structures, biological significance and therapeutic potential. Curr Opin Struct Biol 6: 830-837.

30. Webster GF, Ruggieri MR, McGinley KJ (1981) Correlation of Propionibacterium acnes populations with the presence of triglycerides on nonhuman skin. Appl Environ Microbiol 41: 1269-1270.

31. Nakano K, Kiyokane K, Benvenuto-Andrade G, Gonzalez S (2007) Realtimereflectance confocal microscopy, a noninvasive tool for in vivo quantitative evaluation of comedolysis in the rhino mouse model. Skin Pharmacol Physiol 20: 29-36.

32. Takaoki M, Kawaji H (1980) Impaired antibody response against T-dependent antigens in rhino mice. Immunology 40: 27-32.

33. Mirshahpanah P, Maibach HI (2007) Models in acnegenesis. Cutan Ocul Toxicol 26: 195-202.

34. Shi Y, Elmets CA, Smith JW, Liu YT, Chen YR, et al. (2007) Quantitative proteomes and in vivo secretomes of progressive and regressive UV-induced fibrosarcoma tumor cells: Mimicking tumor microenvironment using a dermisbased cell-trapped system linked to tissue chamber. Proteomics 7: 4589-4600. 